\title{
High Inflammatory Tendency Induced by Malignant Stimulation Through Imbalance of CD28 and CTLA-4/PD-I Contributes to Dopamine Neuron
} Injury

\author{
Li Dong' \\ Yu-Min Zheng ${ }^{2}$ \\ Xiao-Guang Luo ${ }^{3}$ \\ Zhi-Yi He ${ }^{2}$
}

'Department of Neurology, The Fourth Affiliated Hospital of China Medical University, Shenyang, People's Republic of China; ${ }^{2}$ Department of Neurology, The First Affiliated Hospital of China Medical University, Shenyang, People's Republic of China; ${ }^{3}$ Department of Neurology, The First Affiliated Hospital of South University of Science and Technology, The Second Clinical College of Jinan University, Shenzhen People's Hospital, Shenzhen, People's Republic of China
Correspondence: Xiao-Guang Luo Department of Neurology, The First Affiliated Hospital of South University of Science and Technology, The Second Clinical College of Jinan University, Shenzhen People's Hospital, Shenzhen, People's Republic of China

Email guanche537@I26.com

Zhi-Yi He

Department of Neurology, The First Affiliated Hospital of China Medical University, Shenyang, People's Republic of China

Email yizizhan40559@163.com
Background: Parkinson's disease is a common neurodegenerative disease in the elderly. The incidence of various cancers in Parkinson's disease patients is significantly lower than in healthy people. Parkinson's disease patients are individuals with a high tendency for inflammation, whose peripheral immune system is represented in an activated state. We hypothesized that the hyperinflammatory predisposition of Parkinson's disease patients is pathogenic.

Methods: DBA/1 mice were used to simulate "highly inflammatory individuals", and the carcinogen DEN was used to induce malignancy. Hematoxylin \& eosin (H\&E) staining was used to observe the formation of lung tumors. Apoptosis of neurons was observed by terminal deoxynucleotidyl transferase-mediated dUTP nick end labeling (TUNEL) staining. Immunohistochemistry and flow cytometry were used to observe CD4, CD28, major histocompatibility complex II (MHCII), cytotoxic T-lymphocyte-associated antigen 4 (CTLA-4), and programmed death 1 (PD-1). The ionized calcium binding adaptor molecule-1 (IBA-1) + inducible nitric oxide synthase (iNOS) was used to label M1 microglia, and IBA-1 + arginase 1 (Arg1) was used to label M2 microglia by immunofluorescence. The expression of proinflammatory cytokines tumor necrosis factor- $\alpha$ (TNF- $\alpha$ ), interleukin-1 $\beta$ (IL-1 $\beta$ ), and antiinflammatory cytokines IL-10 and IL-4 was detected by ELISA.

Results: DBA/1 mice with high inflammatory tendency showed a continuous increase of peripheral inflammation, promoting intracranial inflammation, decreasing the tumor incidence and increasing the neurodegeneration under induction of malignant change. CD28 and CTLA-4/PD-1 reduced the T-cell-dominated inflammatory response, reduced the intracerebral inflammatory response, protected from neurodegeneration, and increased the incidence of tumor. Combination of CTLA-4 and PD-1 blocker can overactivate T cells, worsen peripheral and intracranial inflammation, reduce the incidence of tumor, cause damage to dopamine neurons, and promote the occurrence of neurodegeneration.

Conclusion: High inflammatory tendency induced by malignant stimulation through the imbalance of CD28 and CTLA-4/PD-1 leads to dopamine neuron injury.

Keywords: high inflammatory tendency, malignant stimulation, CD28, CTLA-4/PD-1, dopamine neuron injury

\section{Introduction}

Parkinson's disease (PD) is a common neurodegenerative disease in the elderly. ${ }^{1,2}$ The epidemiological studies of PD demonstrated that the occurrence of PD is contrary to the incidence of malignant tumors. The incidence of lung cancer, ${ }^{3}$ 
colon cancer, ${ }^{4}$ and other cancers in PD patients is significantly lower than in healthy people, ${ }^{5}$ suggesting that individuals with PD have a tendency to have stronger cancer resistance. On the other hand, cancer is closely related to $\mathrm{PD}$, and some pathogenic genes of $\mathrm{PD}$ are also oncogenes. ${ }^{6}$ Parkin, PINK1, and SNCA are not only pathogenic genes of Parkinson's disease, but also closely related to the incidence of various malignant tumors such as ovarian cancer, lung cancer, and liver cancer. ${ }^{7-10} \mathrm{PD}$ patients were supposed to have a higher risk of malignant transformation, however, actually the incidence of various cancers in PD patients is significantly lower than in healthy people. ${ }^{11,12}$

PD patients are individuals with a high tendency for inflammation, whose peripheral immune system is represented in an activated state. The secretion of inflammatory factors in peripheral blood of PD patients is significantly increased, ${ }^{13}$ multiple inflammation-related receptors are over-expressed on peripheral immune cells, including antigen-presenting protein MHCII. These phenomena suggest the abnormal activation of peripheral immunity in PD patients. Moreover, it has been found that many pathogenic proteins of PD are actively involved in inflammatory pathways and homeostasis of inflammation. ${ }^{14}$ In addition, in the genome sequencing of more than 1000 PD patients, 24 gene loci were detected to be correlated with the risk of PD, among which 14 loci were confirmed to be involved in the regulation of immune inflammation. ${ }^{15}$

We hypothesized that the hyperinflammatory predisposition of PD patients is pathogenic. People with high inflammatory tendency, under stimulation by external malignant change, could produce excessive inflammatory response, preventing the occurrence of tumors, however causing neurodegenerative damage in the brain.

In this study, DBA/1 mice were used to simulate "highly inflammatory individuals", and the carcinogen diethylnitrosamine (DEN) was used for malignant stimulation. $\mathrm{DBA} / 1$ is an inbred mouse and widely used in immune inflammation studies. DBA/1 mice are spontaneously formed of arthritis and are also used to prepare collagen-induced arthritis model. DBA/1 mice are used to establish the inflammation model of central nervous system, having a high tendency of inflammation background. Compared with C57BL/6, the incidence of tumor in DBA/ 1 was reduced, however, the damage of dopaminergic neurons in the brain was significantly increased. Moreover, the T-cell-dominated inflammatory response was enhanced by the administration of CTLA- 4 and PD-
1 inhibitors, leading to a reduced incidence of tumor and enhanced death and neurodegeneration of dopamine neurons. This study demonstrated a close relationship among high inflammation, tumor, and neurodegeneration, as well as regulating inflammation in the outcome of cancer and neurodegeneration.

\section{Materials and Methods Laboratory Animals and Groups}

DBA/1 mice and C57BL/6 mice, SPF grade, male, 6-8 weeks, were purchased from Beijing Vital River Laboratory Animal Technology Co., Ltd. (SCXK (Beijing) 201,200,070). Mice were raised in the Laboratory Animal Department of China Medical University (SYXK (Liao) 201,880,005). The animal experiment was approved by the Ethics Committee of Experimental Animal Welfare of China Medical University (IACUC. No. 20,180,001). Mice were divided into DBA/1 untreated group (DBA) $(\mathrm{n}=10), \mathrm{C} 57 \mathrm{BL} / 6$ untreated group (C57) $(n=10)$, DBA/1 treated with diethylnitrosamine (DEN) group (DD) $(n=10), C 57 \mathrm{BL} / 6$ treated with DEN group $(\mathrm{CD})(\mathrm{n}=10), \mathrm{DBA} / 1+\mathrm{DEN}+$ anti-CTLA -4 and anti-PD-1 combined treatment group (DDCP group), $(\mathrm{n}=10)$ and $\mathrm{C} 57 \mathrm{BL} / 6+\mathrm{DEN}+$ anti-CTLA-4 and anti-PD-1 combined treatment group (CDCP group) $(\mathrm{n}=10)$. Mice in DD and CD groups were subcutaneously injected with $1 \%$ DEN $56 \mathrm{mg} / \mathrm{kg}$ once a week for eight weeks. Mice in DDCP and CDCP groups were treated by intraperitoneal injection of CTLA-4 inhibitor, ipilimumab, and anti-PD-1, durvalumab, $10 \mathrm{mg} / \mathrm{Kg}$ at 7, 8, 9, 12, 15, 18, 21, 24, 27 days after DEN injection. All animal experiment procedures performed in this study were reviewed and approved by the Ethics Committee of Experimental Animal Welfare of China Medical University (IACUC. No. 20,180,001) and followed the National Institutes of Health guide for the care and use of Laboratory animals (NIH Publications No. 8023, revised 1978).

\section{Hematoxylin-Eosin Staining}

HE staining was used to observe the formation of lung tumors. At week 4 and week 8, blood samples were taken from the orbital vein and sera were immediately isolated. Then mice in each group were sacrificed by dislocating their cervical spine. Following that, the lung tissue was collected and fixed with paraformaldehyde. Tissue was dehydrated by ethanol at different concentrations, made transparent with xylene, embedded and block-mounted in 
paraffin. Sections were cut and stained with hematoxylineosin. An OLYMPUS BX-43 was used to capture images.

\section{TUNEL}

Apoptosis of neurons was observed by TUNEL staining. Paraffin-embedded sections of brain tissue were cut into 5 $\mu \mathrm{m}$ sections and treated with $50 \mu \mathrm{L}$ TUNEL reaction solution. The sections were incubated at $37^{\circ} \mathrm{C}$ for 60 min, and then incubated in $50 \mu \mathrm{L}$ streptomycin avidinhorseradish peroxidase solution for $30 \mathrm{~min}$. The nuclei were stained with DAPI; after dehydration, the slices were sealed with an anti-fluorescence quenching agent. The fluorescence was detected under a fluorescence microscope and photographed.

\section{Immunohistochemistry}

Paraffin-embedded substantia nigra pars compacta (SNpc) brain tissue blocks were sectioned at $5 \mu \mathrm{m}$. The citric acid antigen repair buffer was placed in the microwave oven for antigen repair, and the sections were placed in 3\% hydrogen peroxide solution to block endogenous peroxidase. 3\% BSA was added for blocking at room temperature for $30 \mathrm{~min}$. Then anti-CD4 (Abcam, USA, ab183685, Rabbit monoclonal [EPR19514] to CD4), anti-CD28 (Abcam, USA, ab243228, Rabbit monoclonal [EPR22076] to CD28), anti-CTLA-4 (Abcam, USA, ab237712, Rabbit monoclonal [CAL49] to CTLA4), anti-PD-1 (CST, USA, \#84,651, Rabbit monoclonal [D7D5W] to -PD-1) and anti-MHCII (Sigma, USA, SAB4700663, Rat monoclonal [M5/114] to - MHCII) antibodies were separately added to examine the number of infiltrating $\mathrm{T}$ cells in the brain. The tissue sections were covered with the secondary antibody (Abcam, USA, ab150113/ab150077) and incubated at room temperature for $50 \mathrm{~min}$. DBA kit was used for color development, hematoxylin was used to re-stain the nucleus. After gradient alcohol dehydration and sealing, the slices were observed under a microscope. At least 3 slices per mouse in the different groups (10 mice per group, $n=10$ ) were used in the analysis.

\section{Immunofluorescence}

$\mathrm{SNpc}$ tissues from the brain were subjected to deparaffinized, hydrated, antigen repaired $(0.01 \mathrm{M}$ citrate buffer, $\mathrm{pH}$ 6.0) and goat serum closed at $37^{\circ} \mathrm{C}$ for $30 \mathrm{~min}$. Serum was poured out, and $\mathrm{TH}$ antibody was added to determine the survival of dopamine neurons. IBA-1 (Abcam, USA, ab178846, Rabbit monoclonal
[EPR16588] to IBA-1) + iNOS (Abcam, USA, ab178945, Rabbit monoclonal [EPR16635] to iNOS) labeled M1 type microglia and IBA-1 (Abcam, USA, ab178846, Rabbit monoclonal [EPR16588] to IBA-1) + Arg1 (CST, USA, \#93,668, Rabbit monoclonal [D4E3M] to - Arg1) labeled M2 type microglia were incubated at $4{ }^{\circ} \mathrm{C}$ overnight. The sections were washed with PBS and fluorescence-labeled secondary antibody was added. The sections were incubated at $37^{\circ} \mathrm{C}$ in the dark for $30 \mathrm{~min}$ and then washed with PBS. DAPI stained nuclei were added and incubated at room temperature for $10 \mathrm{~min}$. The slices were rinsed with PBS and sealed with anti-fluorescence quenching. Photographs were taken with a fluorescence microscope. At least 3 slices per mouse in the different groups (10 mice per group, $n=10$ ) were used in the analysis.

\section{ELISA Assay}

The expression of pro-inflammatory cytokines TNF- $\alpha$, IL$1 \beta$ and anti-inflammatory cytokines IL-10 and IL-4 in serum and SNpc tissues of brains of mice in each group was detected by ELISA kit. The operation steps were strictly carried out according to the instructions of the kit (Abcam, USA).

\section{Flow Cytometry}

Mice were narcotized, blood was collected from the abdominal aorta, and EDTA anticoagulant was added. Blood lymphocytes of mice were isolated by blood lymphocyte separation kit, the second layer of milky white lymphocytes was carefully moved to another tube. After centrifugation, the collected lymphocytes were resuspended with PBS, and antibodies of CD4 (Abcam, USA, ab183685, Rabbit monoclonal [EPR19514] to CD4), CD28 (Abcam, USA, ab243228, Rabbit monoclonal [EPR22076] to CD28), CTLA-4 (Abcam, USA, ab237712, Rabbit monoclonal [CAL49] to CTLA4), PD-1 (CST, USA, \#84,651, Rabbit monoclonal [D7D5W] to -PD-1), and MHCII (Sigma, USA, SAB4700663, Rat monoclonal [M5/114] to - MHCII) were added respectively for flow cytometry .

\section{Statistical Analysis}

SPSS 19.0 statistical software (IBM) was used for statistical analysis. The experimental data were expressed as mean \pm standard deviation. The results of the experiments were analyzed using the $\chi 2$ test for ratio rate comparison 
and one-way analysis of variance for the other comparisons. $\mathrm{P}<0.05$ was considered to be statistically significant.

\section{Results}

\section{DBA/I Mice with High Inflammatory} Tendency Showed Decreased Tumor Incidence and Continuous Increasing of Peripheral Inflammation

After carcinogenic induction, the tumor incidence of DBA/ 1 mice was decreased significantly compared with C57BL/ 6. HE staining showed that tumors were observed in mice in both $\mathrm{CD}$ and DD groups (Figure 1A). After eight weeks' treatment with DEN, the tumor incidence was about $50 \%$ in DD group, and $100 \%$ in CD group. It suggested that the incidence of tumor was reduced in mice with high inflammatory tendency after malignant transformation. Moreover, after DEN induction, the proinflammatory factors TNF- $\alpha$ and IL- $1 \beta$ in peripheral blood of DBA/1 mice were also increased, the anti-inflammatory factors IL-10 and IL-4 were persistently decreased. Interestingly, in C57BL/6 mice, TNF- $\alpha$ and IL-1 $\beta$ were increased first and then decreased, IL-10 and IL-4 were decreased first and then increased (Figure 1B).
DBA/I Mice with High Inflammatory

Tendency Showed Promotion of Intracranial Inflammation and Increased Neurodegeneration Under Induction of Malignant Change

After carcinogenic induction, microglia in the brain of DBA/1 mice were significantly activated. M1 type microglia were dominant in DBA/1 mice, M2 type microglia were relatively reduced. Moreover, M1 type microglia were significantly increased in DD group compared with the control group. In this study, IBA-1 + iNOS was used to label M1 type microglia, and IBA-1 + Arg1 was used to label M2 type microglia by immunofluorescence, and the results showed that the activation of microglia in DBA/1 mice was more than that in C57BL/6 mice (Figure $2 \mathrm{~A})$. The damage of dopamine neurons in DBA/1 mice was more significant than that in C57BL/6 mice under DEN induction. TH staining showed that the number of dopamine neurons in DBA/1 mice was significantly reduced compared with that in C57BL/6 mice (Figure 2B). Tunel staining assay demonstrated that the apoptosis of substantia nigra neurons of DBA/1 mice was higher than that of C57BL/6 mice (Figure 2C).
A

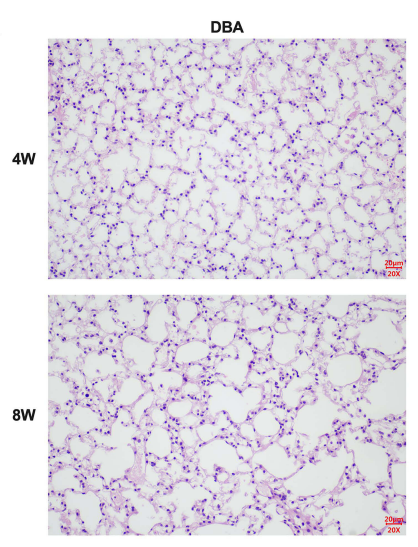

B

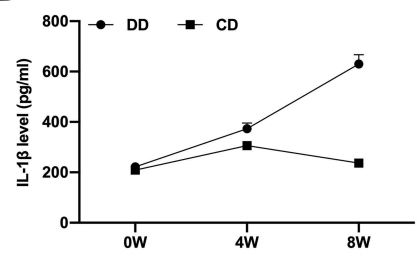

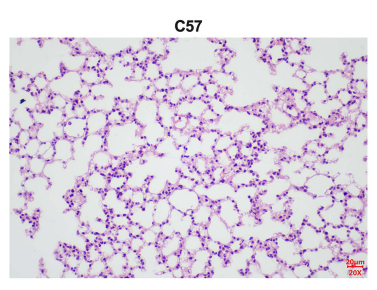
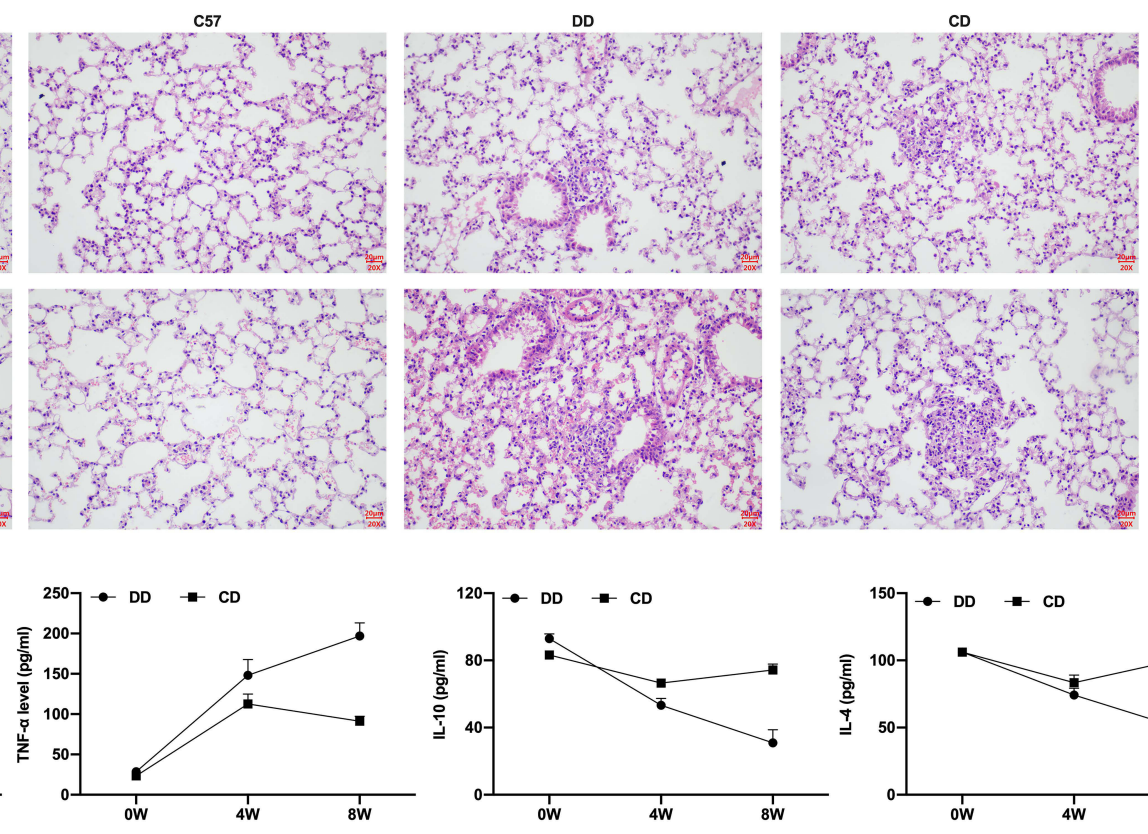
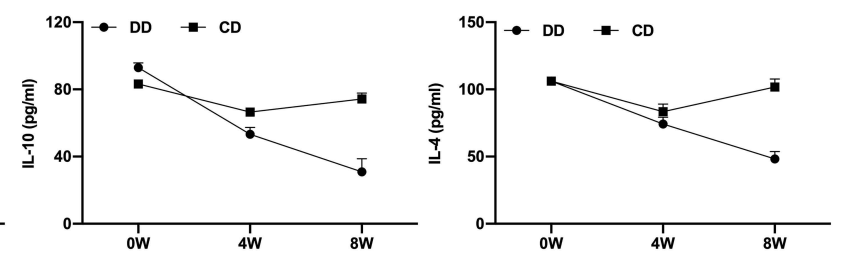

Figure I Comparison of the tumor incidence and peripheral inflammation of mice in different groups. The results displayed that tumors were observed in lung tissue of mice in both CD and DD groups (A). The pro-inflammatory factors TNF- $\alpha$ and IL-I $\beta$ in peripheral blood of DBA/I mice were also increased, the anti-inflammatory factors IL- 10 and IL-4 were persistently decreased, in C57BL/6 mice, TNF- $\alpha$ and IL-I $\beta$ were increased firsty and then decreased, IL-I0 and IL-4 were decreased firsty and then increased (B). $n=10$. 
A
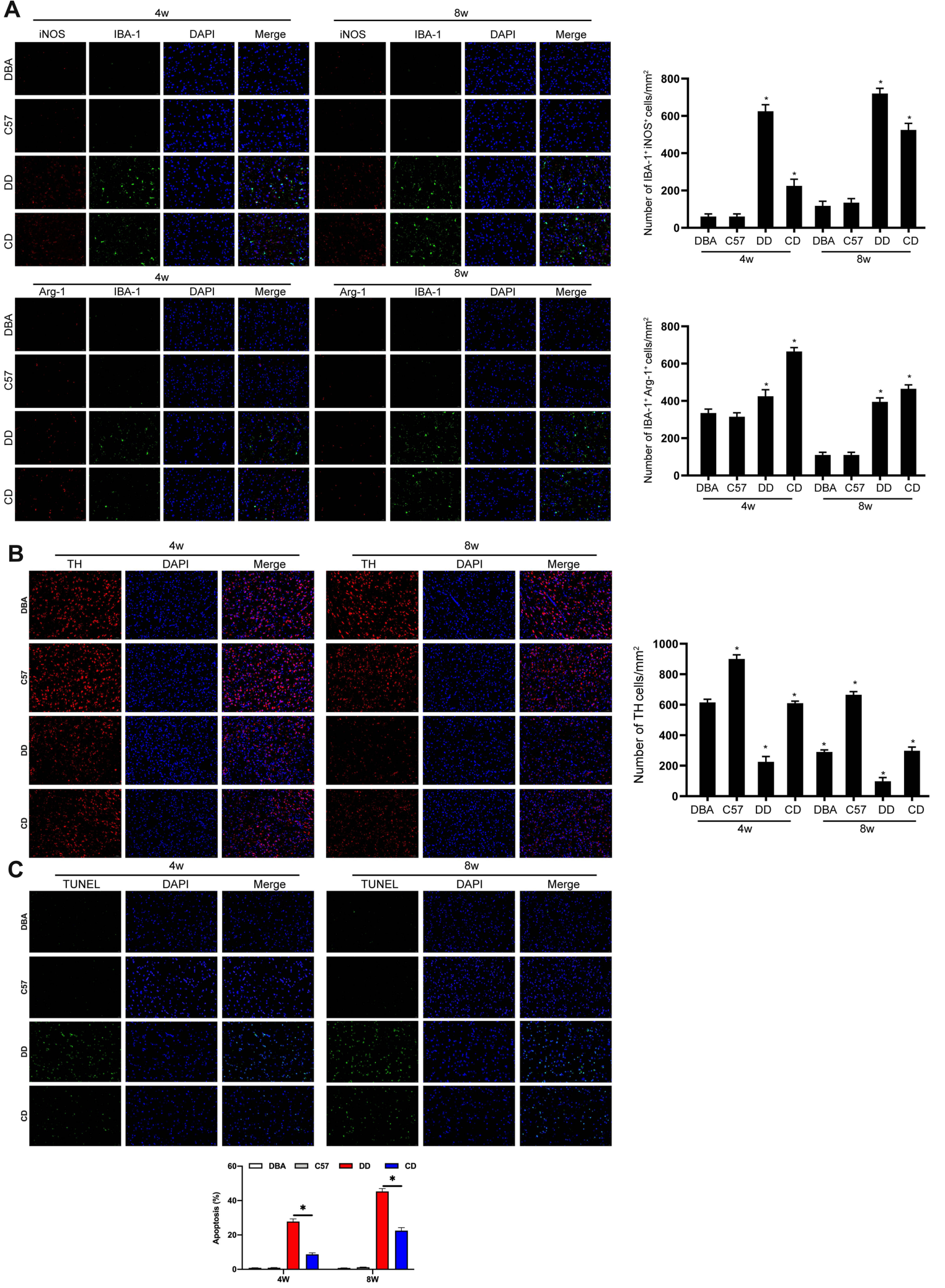

Figure 2 Comparison of the intracranial inflammation and neurodegeneration of mice in different groups. The results displayed that MI type microglia were dominant in DBA/I mice, M2 type microglia were relatively reduced, $M 1$ type microglia were significantly increased in DD group compared with the control group, and the activation of microglia in SNpc tissues of DBA/I mice was more than that in C57BL/6 mice (A). TH staining showed that the number of dopamine neurons in DBA/I mice was significantly reduced compared with that in C57BL/6 mice (B). Tunel staining assay demonstrated that the apoptosis of substantia nigra neurons of DBA/I mice was higher than that of $\mathrm{C} 57 \mathrm{BL} / 6$ mice $(\mathbf{C})$. *Indicated $P<0.05 . \mathrm{n}=10$. 


\section{CD28 and CTLA-4/PD-I Reduced the} T-Cell-Dominated Inflammatory

Response, Reduced Intracerebral Inflammatory Response, and Protected Against Neurodegeneration

Peripheral $\mathrm{T}$ cells were activated and intracranial inflammatory response was significantly increased in the DD group. The flow cytometry showed that CD4, CD28, and MHCII in peripheral blood lymphocytes of DD group were significantly increased, while CTLA-4 and PD-1 were significantly decreased (Figure 3A). Th1-mediated TNF- $\alpha$ and IL- $1 \beta$ levels in the DD group continued to increase, however, Th2-mediated IL-10 and IL-4 levels decreased continually (Figure $3 \mathrm{~B}$ ). T cells' activation in the DBA/1 group was significantly increased, and the expressions of CTLA-4 and PD-1 were gradually decreased. To detect $\mathrm{T}$ cell number in mouse brain under DEN stimulation, the expression of CD4, CD28, MHCII, CTLA-4 and PD-1 were measured by immunohistochemical staining (Figure 3C). The results showed that the expressions of CD4, CD28, and MHCII in the brain increased gradually with time, while the expressions of CTLA-4 and PD-1 decreased over time in DBA/1 mice compared with $\mathrm{C} 57 \mathrm{BL} / 6$ mice. These results suggest that activated $\mathrm{T}$ cells participate and worsen intracranial inflammation.

\section{Combination of CTLA-4 and PD-I Blocker Can Overactivate T Cells, Worsen Peripheral and Intracranial Inflammation, and Reduce the Incidence of Tumor}

The combination of CTLA-4 and PD-1 blockers can reduce the incidence of tumors. DBA/1 mice were treated with CTLA-4 and PD-1 blocker after injection of DEN, and the tumor incidence at 8 weeks' treatment was reduced to $50 \%$ compared with DD group, while the tumor incidence in C57BL/6 mice was about 20\%. The combination of CTLA-4 and PD-1 blockers can promote the activation of $\mathrm{T}$ cells. The results showed the levels of CD4, CD28, MHCII in peripheral lymphocytes were significantly increased in DDCP group and CDCP group compared to that in the $\mathrm{DD}$ and $\mathrm{CD}$ groups, however, the expression of CTLA 4, PD-1 was significantly reduced (Figure 4A), the changes in the DDCP group were the most evident. Furthermore, immunohistochemistry results demonstrated similar results, ie, that the expression of CD4, CD28 and MHCII in mouse brain tissues was increased, while the expression of CTLA-4 and PD1 was decreased in the DDCP group and CDCP group compared to that in the DD and CD groups (Figure 4B). Furthermore, ELISA results showed that CTLA-4 and PD-1 blockers could activate the inflammatory response after malignant stimulation in DBA/1, with increased TNF- $\alpha$ verify and IL- $1 \beta$ levels and decreased IL-10 and IL-4 levels (Figure 4C).

\section{Combination of CTLA-4 and PD-I Blocker Can Cause Damage to Dopamine Neurons, Promote the Occurrence of Neurodegeneration}

Under the combination treatment of CTLA-4 and PD-1 blockers, brain microglia were activated and dopaminergic neurons were damaged. Immunofluorescence showed that CTLA-4 and PD-1 blockers activated microglial activity in DBA/1 under DEN stimulation, and M1 type microglia were increased, M2 type microglia were decreased (Figure 5A). At the same time, compared with the DD group, the number of dopamine neurons in the DDCP group was decreased (Figure 5B), and the apoptosis rate of substantia nigra neurons was increased (Figure 5C).

\section{Discussion}

Parkinson's disease is a common neurodegenerative disease. However, its etiology and pathogenesis are still unclear. PD is considered to be the result of the interaction of multiple factors. In this study, we demonstrated that harmful stimulation induced in individuals with a high tendency for inflammation activates the $\mathrm{T}$ cells' immune response, increases the stimulating factor, decreases the inhibitory factor, leading to excessive inflammation spreading to the brain, eventually leading to the damage of neurons. Because the co-inhibitory molecules CTLA-4 and $\mathrm{PD}-1$ on $\mathrm{T}$ cells can down-regulate the immune response, we inhibited the expression of CTLA-4 and PD-1, and found that $\mathrm{T}$ cells were activated. Although the occurrence of tumor was reduced, the inflammatory response and the degenerative injury of neurons were aggravated in the brain. The results suggested that a body with a high inflammatory tendency is at high risk of PD, and the disorder of immune mechanism may be one of the important factors of PD. 
A
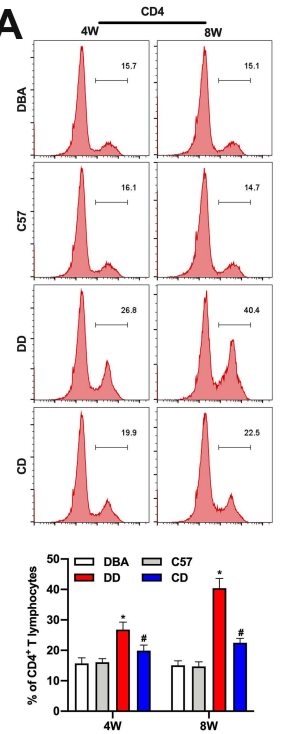

B
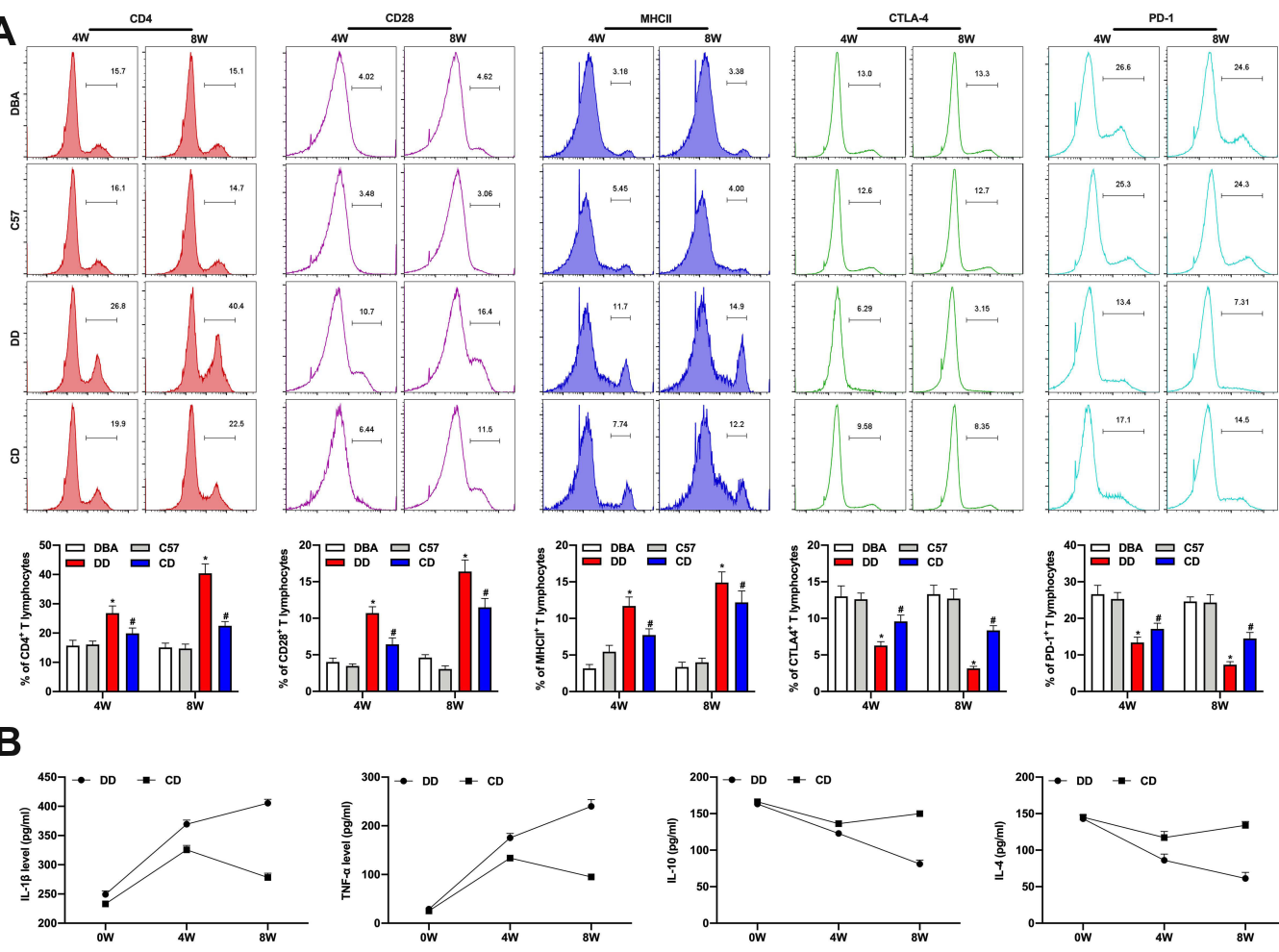
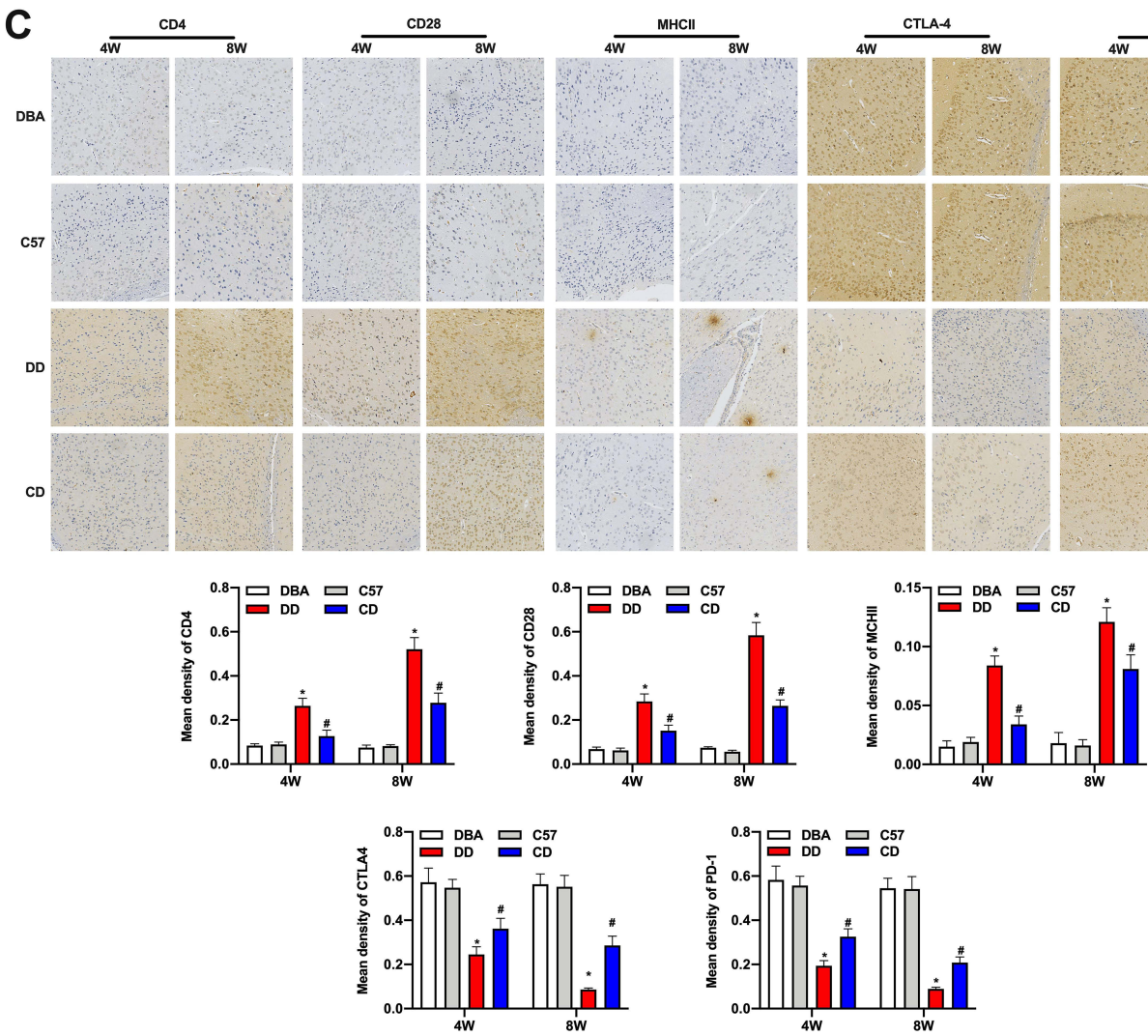

Figure 3 Comparison of the intracerebral inflammatory response and neurodegeneration of mice in different groups. The flow cytometry showed that CD4, CD28, and MHCII in peripheral blood lymphocyte gate of DD group were significantly increased, while CTLA-4 and PD-I were significantly decreased (A). Th I-mediated TNF- $\alpha$ and ILI $\beta$ levels in the DD group continued to increase, however, Th2-mediated IL-I0 and IL-4 levels decreased continually in serum of the mice (B). Immunohistochemical staining showed that the expressions of CD4, CD28, and MHCII in the SNpc tissues of brain increased gradually with time, while the expression of CTLA-4 and PD-I decreased over time in DBA/I mice compared to C57BL/6 mice (C). *Indicated $P<0.05$ compared with DBA group; ${ }^{\#}$ Indicated $P<0.05$ compared with C57 group5. $n=10$. 

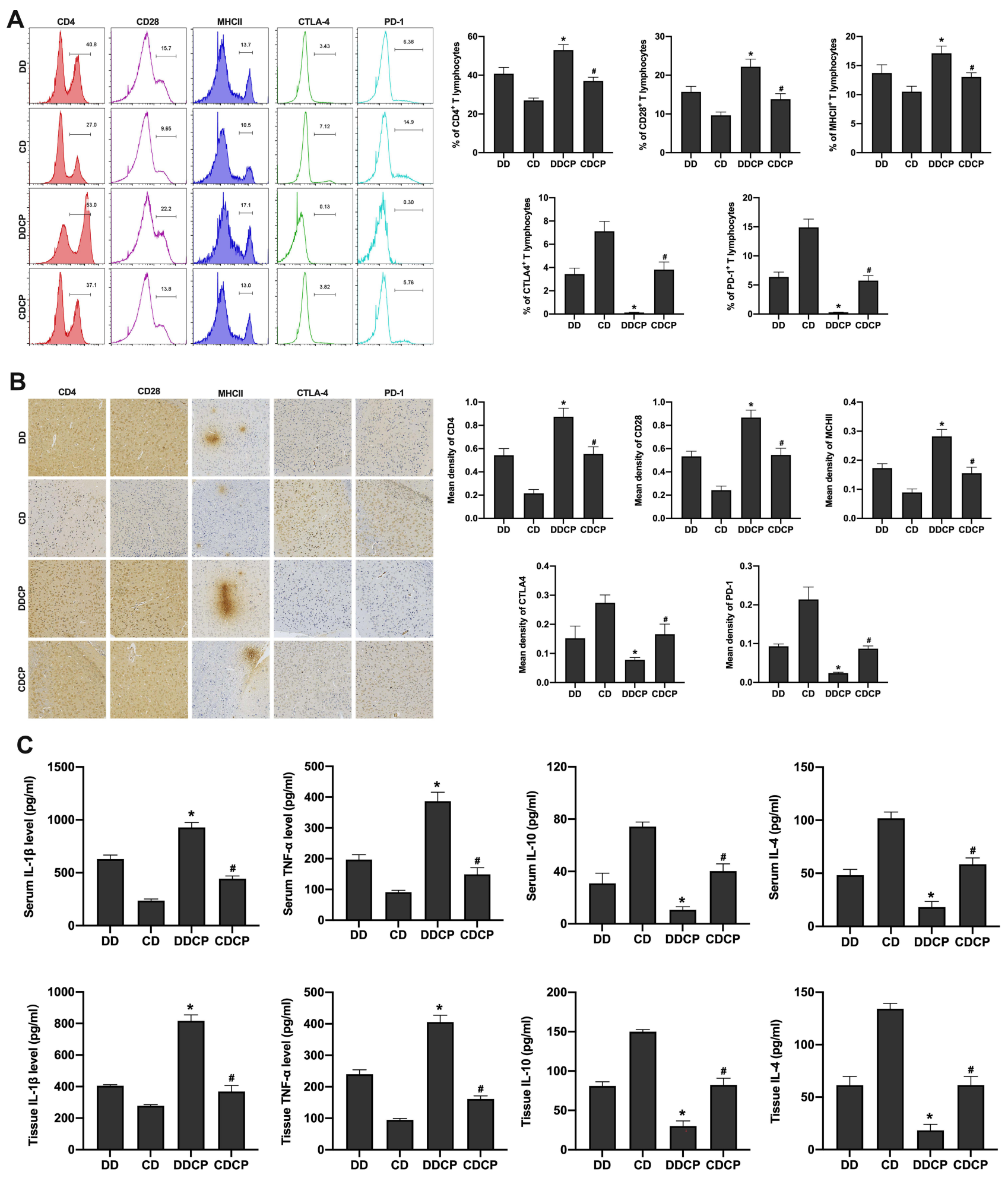

Figure 4 Combination of activated T cells, peripheral and intracranial inflammation and the incidence of tumor among CTLA-4 and PD-I blocker treated mice. The levels of CD4, CD28, MHCll in peripheral lymphocyte gate were significantly increased in DDCP group and CDCP group compared to that in the DD and CD group, however, the expression of CTLA 4, PD-I was significantly reduced (A). Immunohistochemistry results demonstrated similar results, ie, that the expression of CD4, CD28, and MHCII in mice SNpc tissues was increased, while the expression of CTLA-4 and PDI was decreased in the DDCP group and CDCP group compared to that in the DD and CD group (B). ELISA results showed that CTLA-4 and PD-I blockers could activate the inflammatory response after malignant stimulation in DBA/I, with increased TNF- $\alpha$ verify and

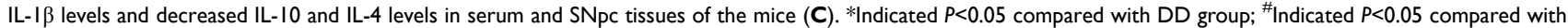
$C D$ group. $n=10$. 

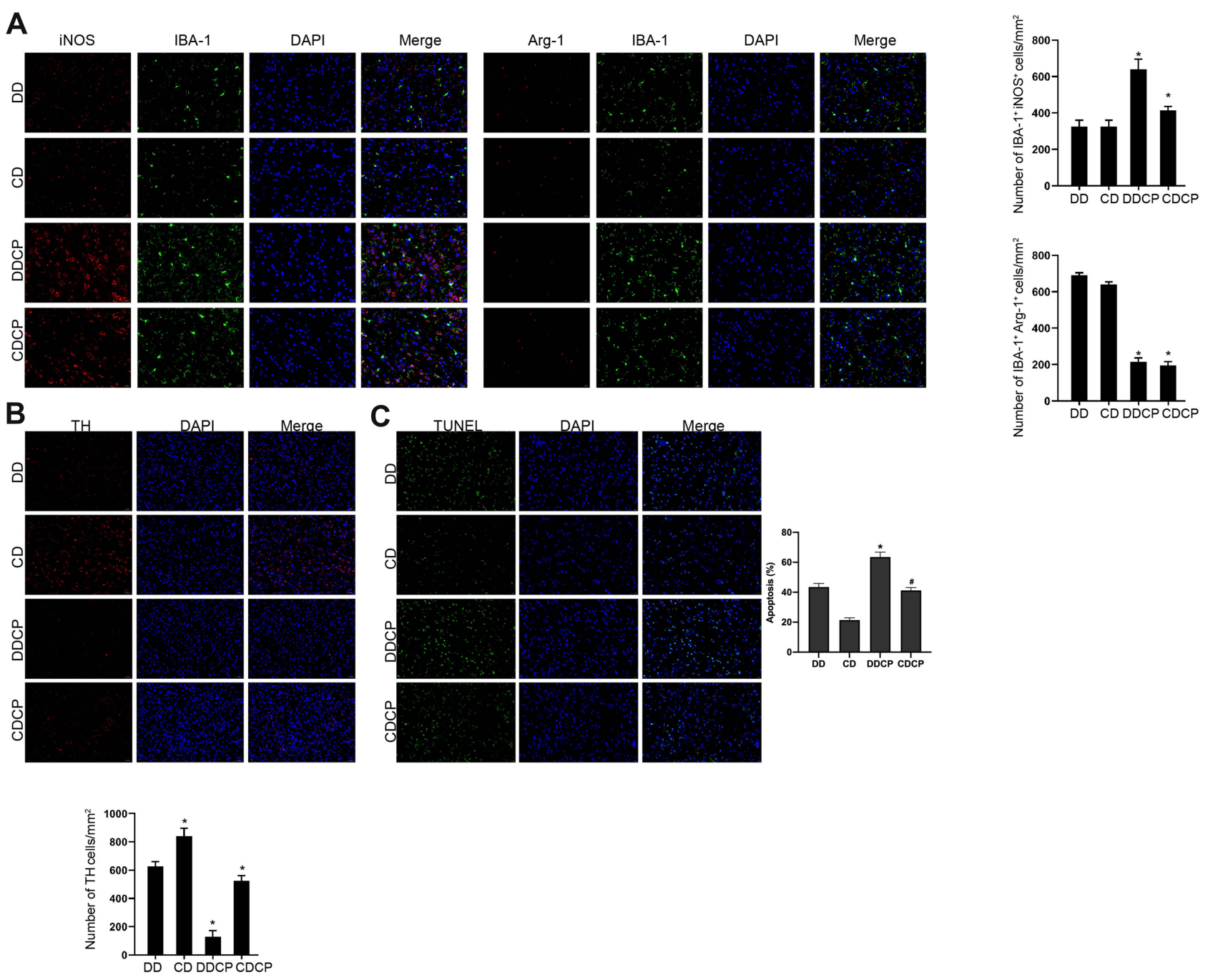

Figure 5 Combined damage to dopamine neurons and the occurrence of neurodegeneration among CTLA-4 and PD-I blocker treated mice. Immunofluorescence showed that CTLA-4 and PD-I blockers activated microglial activity in DBA/I under DEN stimulation, and MI type microglia were increased, M2 type microglia were decreased (A). TH staining showed that compared with the DD group, the number of dopamine neurons in the DDCP group was decreased (B). Tunel staining assay demonstrated that compared with the DD group, the apoptosis rate of substantia nigra neurons was increased in the DDCP group (C). *Indicated $P<0.05$ compared with DD group; ${ }^{*}$ Indicated $P<0.05$ compared with $C D$ group. $n=10$.

\section{As Parkinson's disease is featured as} a neurodegenerative disorder and is closely correlated with cancer development, we explored the inflammatory tendency and immune mechanism in the association of Parkinson's disease and tumorigenesis incidence. In this study, under malignant stimulation, the incidence of tumor in $\mathrm{DBA} / 1$ mice was lower than that in $\mathrm{C} 57 \mathrm{BL} / 6$ mice. Additionally, the peripheral pro-inflammatory cytokines TNF- $\alpha$ and IL-1 $\beta$ were continuously increased, the antiinflammatory cytokines IL-10 and IL-4 were decreased, and the degeneration and damage of neurons in the brain were significant. In $\mathrm{C} 57 \mathrm{BL} / 6$ mice, TNF- $\alpha$ and IL- $1 \beta$ reached the highest levels at the fourth week after stimulation and then gradually decreased. We considered that after four weeks' stimulation, the inflammatory response in wild mice was initiated to down-regulated. However, in DBA/1 mice the inflammatory response was gradually increased, suggesting that inflammation in "high-inflammation mice" was deregulated, eventually leading to apoptosis and damage of dopamine neurons. Clinically, high levels of IL-1 $\beta$, TNF- $\alpha$ have been reported in the serum and cerebrospinal fluid of PD patients. ${ }^{16}$ This is consistent with our results, thus it is considered that inflammatory factors may also be involved in the occurrence of central inflammation leading to the damage of dopamine neurons. We considered that the tendency of high inflammation in the body is a double-edged sword, which not only inhibits tumorigenesis, but also initiates neuronal damage in the brain. The 
pathogenesis of PD is closely related to inflammatory response. Combined with the significantly decreased tumor incidence in PD patients, we speculate that this is partially because of PD patients' high inflammatory tendency in the body, ie, the body's inflammatory response is induced when facing malignant stimulation environment, however the inflammation is out of control, leading to the death of dopaminergic neurons in the brain.

In this study, under DEN induction, followed by CTLA-4 and PD-1 inhibitor treatment, CD4, CD28, and MHCII in peripheral and intracranial of DBA/1 mice were significantly increased, CTLA-4 and PD1 were significantly decreased, and intracranial pro-inflammatory factors were significantly increased. These results suggested that malignant stimulation could overactivate the $\mathrm{T}$ cells in highly inflammatory mice and simultaneously participate in and worsen the intracranial inflammatory response, leading to the occurrence of neurodegenerative injury. $T$ cell activation has always been the research area of antitumor immunity and tumor vaccine research and $\mathrm{T}$ cell activation is also closely related to PD. Saunders et al demonstrated that the imbalance between memory $T$ cells and regulatory $\mathrm{T}$ cells was closely related to the severity of PD. ${ }^{17}$ T cell over-activation is also involved in exacerbating inflammation in the brain. In the studies of PD patient brain ${ }^{18}$ and PD animal models, ${ }^{19}$ a large number of $\mathrm{CD}^{4+}$ and $\mathrm{CD}^{8+} \mathrm{T}$ cells were infiltrated. In addition, various treatments in activating $\mathrm{T}$ cell immunity to inhibit tumor growth $^{20}$ also cause autoimmune encephalitis, ${ }^{21}$ transverse myelitis. ${ }^{22}$ These studies suggest that the over-activated $\mathrm{T}$ cell immune system in peripheral blood can cause inflammation of the central nervous system. Therefore, we considered that in "highly inflammatory individuals", T-cell immunity is activated in order to combat malignant stimuli, triggering strong peripheral inflammation, inhibiting tumor, however, promoting inflammatory response and neurodegeneration in the brain.

In this study, under DEN induction, followed by CTLA-4 and PD-1 inhibitor treatment, the tumor incidence rate in DBA/1 mice was decreased, CD28 expression in peripheral blood and brain was increased, and the expression of CTLA-4 and PD1 was further decreased. The expression of pro-inflammatory factors in peripheral blood and brain tissue was also increased, and the expression of anti-inflammatory factors was decreased, therefore the damage and apoptosis of dopamine neurons in the brain were more significant. It was suggested that the combined application of CTLA-4 and PD-1 inhibitor further promoted $\mathrm{T}$ cells' activation, aggravated the damage of dopamine neurons, and significantly decreased the incidence of tumor. These results showed that CTLA-4 and PD-1 could regulate the process of $\mathrm{T}$ cell activation and resist tumor incidence, and aggravate dopamine neuron injury. The activation of $\mathrm{T}$ cells requires dual signals, the first signal comes from the specific binding of MHCantigen complex of Antigen-presenting cells (APC) with $\mathrm{T}$ cell receptor (TCR), and the second signal comes from the binding of co-stimulating molecules (CD28) or coinhibiting molecules (CTLA-4 and PD-1) on the surface of $\mathrm{T}$ cells with the ligands on the APC. CD28, a costimulatory molecule on the surface of T cells, can activate $\mathrm{T}$ cells and promote inflammation after stimulation. The co-inhibitory molecules CTLA- 4 and PD- 1 can inhibit the activation of inflammatory T cells. CD28, CTLA-4, and $\mathrm{PD}-1$ can precisely regulate the transitioning of $\mathrm{T}$ cells to pro-inflammatory Th1 or anti-inflammatory Th2, and affect the activation state of peripheral immunity. The inhibitor of CTLA-4 has shown good treatment efficacy in rheumatoid arthritis, ${ }^{23}$ type 1 diabetes, ${ }^{24}$ and kidney transplant immune system rejection. ${ }^{25}$ On the contrary, coinhibitory molecules such as CTLA-4 and PD-1 are increasingly expressed in tumors, which inhibit the activation of $\mathrm{T}$ cells and weaken the immune surveillance of tumors, becoming the most important mechanism for tumor-cell escape. ${ }^{26}$ This study showed that the combination of CTLA- 4 and PD- 1 inhibitors can activate T cells in highly inflammation-prone animals and promote excessive inflammation, and increase the transformation of microglia into M1 type cells to aggravate the damage of dopamine neurons, promoting the development of $\mathrm{PD}$, however, reducing tumor escape and significantly decreasing the incidence of tumor.

These results demonstrated that individuals with high inflammatory tendency are the basis for the occurrence of Parkinson's disease. When exposed to harmful stimuli, inflammation is initiated to protect the body from the occurrence of malignant tumors. However, due to their high inflammatory tendency, excessive inflammation is caused and eventually dopamine neurons are injured. The harmful stimulus may not only be limited to DEN, it may also include a variety of microbial infections, chemicals, all kinds of physical and chemical stimulations, etc. These harmful stimuli induce excessive inflammation in the individual with high tendency, accumulating gradually over a long period, leading to damage of the central nervous system. This study confirmed that a body with a high 
inflammatory tendency is at high risk of excessive inflammatory response to malignant stimulation. In the process, CTLA-4 and PD-1 as important regulatory factors could reduce the inflammatory response. The blocking of CTLA4 and PD-1 together leads to deterioration of inflammation, increasing the damage to neurons in the brain. Although it could curb the occurrence of tumors, it increases the risk of nerve degeneration. These findings confirmed that a genetic high inflammatory tendency is the pathogenesis of PD risk factors.

Moreover, this investigation indicates the reciprocal negative regulation between the development of tumors and Parkinson's disease, an inflammatory disorder that involves a $\mathrm{T}$ cell-mediated autoimmune response. The negative association has been previously revealed for cancer and other autoimmune disorders, such as scleroderma and myositis. ${ }^{27}$ Antibody therapeutics and immunoregulation have been proven to enhance the prognosis of refractory solid cancer patients but may induce autoimmune effects. $^{28}$ The specific correlation of cancer with other autoimmune diseases should be evaluated in future studies. Meanwhile, it has been identified that immune system dysfunction plays essential roles in the development of PD. Multiple factors, such as immunogenetic regulators, peripheral inflammation mechanism, and autoimmune mechanism, are involved in the modulation of PD. ${ }^{29}$ The association of these factors and other immune regulatory mechanisms with cancer initiation and progression needs to be confirmed by further exploration.

\section{Conclusion}

High inflammatory tendency induced by malignant stimulation through imbalance of CD28 and CTLA-4/PD-1 leads to dopamine neuron injury. Our findings provide novel insight into the understanding of the negative correlation of cancer occurrence and PD-related neuron injury, elucidating the experimental basis of the knowledge of inflammatory tendency and immune mechanisms in the modulation of PD and tumorigenesis. The clinical relationship of PD and cancers should be evaluated in future investigations and more potential mechanisms of the interplay between PD and tumorigenesis should be explored.

\section{Funding}

This research was supported by the National Nature Science Foundation of China (grant no. 81771375).

\section{Disclosure}

The authors declare that they have no competing interests.

\section{References}

1. Hayes MT. Parkinson's disease and parkinsonism. Am J Med. 2019;132(7):802-807. doi:10.1016/j.amjmed.2019.03.001

2. Jankovic J, Tan EK. Parkinson's disease: etiopathogenesis and treatment. J Neurol Neurosurg Psychiatry. 2020;91(8):795-808. doi:10.1136/jnnp-2019-322338

3. Xie X, Luo X, Xie M, Liu Y, Wu T. Risk of lung cancer in parkinson's disease. Oncotarget. 2016;7(47):77319-77325. doi:10.18632/ oncotarget.12964

4. Xie X, Luo X, Xie M. Association between parkinson's disease and risk of colorectal cancer. Parkinsonism Relat Disord. 2017;35:42-47. doi:10.1016/j.parkreldis.2016.11.011

5. Tacik P, Curry S, Fujioka S, et al. Cancer in parkinson's disease. Parkinsonism Relat Disord. 2016;31:28-33. doi:10.1016/j. parkreldis.2016.06.014

6. Espay AJ, Kalia LV, Gan-Or Z, et al. Disease modification and biomarker development in parkinson disease: revision or reconstruction? Neurology. 2020;94(11):481-494. doi:10.1212/ WNL.0000000000009107

7. Mehdi SJ, Ali A, Rizvi MM. Parkin gene alterations in ovarian carcinoma from northern Indian population. Pathol Oncol Res. 2011;17(3):579-586. doi:10.1007/s12253-010-9351-x

8. Picchio MC, Martin ES, Cesari R, et al. Alterations of the tumor suppressor gene parkin in non-small cell lung cancer. Clin Cancer Res. 2004;10(8):2720-2724. doi:10.1158/1078-0432. CCR-03-0086

9. Fujiwara M, Marusawa $\mathrm{H}$, Wang $\mathrm{HQ}$, et al. Parkin as a tumor suppressor gene for hepatocellular carcinoma. Oncogene. 2008;27 (46):6002-6011. doi:10.1038/onc.2008.199

10. Morris LG, Veeriah S, Chan TA. Genetic determinants at the interface of cancer and neurodegenerative disease. Oncogene. 2010;29 (24):3453-3464. doi:10.1038/onc.2010.127

11. Wahabi K, Perwez A, Rizvi MA. Parkin in parkinson's disease and cancer: a double-edged sword. Mol Neurobiol. 2018;55 (8):6788-6800. doi:10.1007/s12035-018-0879-1

12. Ejma M, Madetko N, Brzecka A, et al. The links between parkinson's disease and cancer. Biomedicines. 2020;8(10):10. doi:10.3390/ biomedicines 8100416

13. Wang XM, Zhang YG, Li AL, et al. Relationship between levels of inflammatory cytokines in the peripheral blood and the severity of depression and anxiety in patients with parkinson's disease. Eur Rev Med Pharmacol Sci. 2016;20(18):3853-3856.

14. Dzamko N, Geczy CL, Halliday GM. Inflammation is genetically implicated in parkinson's disease. Neuroscience. 2015;302:89-102. doi:10.1016/j.neuroscience.2014.10.028

15. Nalls MA, Saad M, Noyce AJ, et al. Genetic comorbidities in parkinson's disease. Hum Mol Genet. 2014;23(3):831-841. doi:10.1093/ $\mathrm{hmg} / \mathrm{ddt} 465$

16. Dobbs RJ, Charlett A, Purkiss AG, Dobbs SM, Weller C, Peterson DW. Association of circulating TNF-alpha and IL-6 with ageing and parkinsonism. Acta Neurol Scand. 1999;100(1):34-41. doi:10.1111/j.1600-0404.1999.tb00721.x

17. Saunders JA, Estes KA, Kosloski LM, et al. CD4+ regulatory and effector/memory $\mathrm{T}$ cell subsets profile motor dysfunction in PARKINSON'S disease. $J$ Neuroimmune Pharmacol. 2012;7 (4):927-938. doi:10.1007/s11481-012-9402-z

18. Brochard V, Combadiere B, Prigent A, et al. Infiltration of CD4+ lymphocytes into the brain contributes to neurodegeneration in a mouse model of parkinson disease. J Clin Invest. 2009;119 (1):182-192. doi:10.1172/JCI36470 
19. Benner EJ, Banerjee R, Reynolds AD, et al. Nitrated alpha-synuclein immunity accelerates degeneration of nigral dopaminergic neurons. PLoS One. 2008;3(1):e1376. doi:10.1371/journal.pone.0001376

20. Galaine J, Borg C, Godet Y, Adotevi O. Interest of tumor-specific CD4 T helper 1 cells for therapeutic anticancer vaccine. Vaccines. 2015;3(3):490-502. doi:10.3390/vaccines3030490

21. Williams TJ, Benavides DR, Patrice KA, et al. Association of autoimmune encephalitis with combined immune checkpoint inhibitor treatment for metastatic cancer. JAMA Neurol. 2016;73(8):928-933. doi:10.1001/jamaneurol.2016.1399

22. Liao B, Shroff S, Kamiya-Matsuoka C, Tummala S. Atypical neurological complications of ipilimumab therapy in patients with metastatic melanoma. Neuro Oncol. 2014;16(4):589-593. doi:10.1093/ neuonc/nou001

23. Keystone E, Alkhalaf A, Makkawy M. Subcutaneous abatacept in rheumatoid arthritis: current update. Expert Opin Biol Ther. 2015;15 (8):1221-1230. doi:10.1517/14712598.2015.1065248

24. Orban T, Bundy B, Becker DJ, et al. Costimulation modulation with abatacept in patients with recent-onset type 1 diabetes: follow-up 1 year after cessation of treatment. Diabetes Care. 2014;37 (4):1069-1075. doi:10.2337/dc13-0604
25. Del Bello A, Marion O, Milongo D, Rostaing L, Kamar N. Belatacept prophylaxis against organ rejection in adult kidney-transplant recipients. Expert Rev Clin Pharmacol. 2016;9(2):215-227. doi:10.1586/17512433.2016.1112736

26. Baksh K, Weber J. Immune checkpoint protein inhibition for cancer: preclinical justification for CTLA-4 and PD-1 blockade and new combinations. Semin Oncol. 2015;42(3):363-377. doi:10.1053/j. seminoncol.2015.02.015

27. Giat E, Ehrenfeld M, Shoenfeld Y. Cancer and autoimmune diseases. Autoimmun Rev. 2017;16(10):1049-1057. doi:10.1016/j.autrev.2017. 07.022

28. Yasunaga M. Antibody therapeutics and immunoregulation in cancer and autoimmune disease. Semin Cancer Biol. 2020;64:1-12. doi:10.1016/j.semcancer.2019.06.001

29. Tan EK, Chao YX, West A, Chan LL, Poewe W, Jankovic J. Parkinson disease and the immune system - associations, mechanisms and therapeutics. Nat Rev Neurol. 2020;16(6):303-318. doi:10.1038/ s41582-020-0344-4
Journal of Inflammation Research

\section{Publish your work in this journal}

The Journal of Inflammation Research is an international, peerreviewed open-access journal that welcomes laboratory and clinical findings on the molecular basis, cell biology and pharmacology of inflammation including original research, reviews, symposium reports, hypothesis formation and commentaries on: acute/chronic inflammation; mediators of inflammation; cellular processes; molecular

\section{Dovepress}

mechanisms; pharmacology and novel anti-inflammatory drugs; clinical conditions involving inflammation. The manuscript management system is completely online and includes a very quick and fair peerreview system. Visit http://www.dovepress.com/testimonials.php to read real quotes from published authors. 